\title{
EL DERECHO A LA EDUCACIÓN: UN DERECHO FUNDAMENTAL EN COLOMBIA ${ }^{1}$
}

\author{
THE RIGHT TO EDUCATION: A FUNDAMENTAL RIGHT IN COLOMBIA
}

\begin{abstract}
"La educación no es un bien estático que debamos contemplar fuera de su contexto general; es un proceso en curso y posee un valor intrínseco en tanto que derecho humano. La gente no solo tiene derecho a recibir una educación de calidad ahora, sino también a adquirir las competencias y el conocimiento necesarios para asegurar el reconocimiento y el respeto a largo plazo de todos los derechos humanos." (Vernor Muñoz, 2006. En: UNICEF: Un enfoque de la educación basado en los derechos humanos)
\end{abstract}

John Fernando Restrepo Tamayo

Doctor en derecho

Profesor de la Universidad de Medellín - Colombia

\begin{abstract}
Resumo
O objetivo deste texto é apresentar as várias expressões sóciojurídicas e constitucionais nas quais o direito à educação na Colômbia foi concebido. Isso supõe uma ênfase especial na transição que foi feita da educação como um serviço público ou como um direito de benefício a ser concebido e descrito como um direito fundamental, além da ordem lexicográfica. A razão de se conceber como direito fundamental é condicionada por três aspectos: o bloco constitucional; a interpretação abrangente da ordem jurídica que reconhece a educação como um direito fundamental, não apenas a favor das crianças, e a realização dos princípios de dignidade e autodeterminação. Para servir a esse fim, será apresentada primeiro uma maneira de entender as particularidades normativas que condicionam a categorização de um direito como fundamental; a educação então será inserida nessa concepção de direito e, finalmente, as principais tensões que surgiram no cenário político colombiano serão desenvolvidas a partir dos significados que a Constituição de 1991 historicamente apresentou sobre o direito à educação e expressada em dois setores particulares da interpretação jurisprudencial do direito à educação.
\end{abstract}

Palavras-chave: educação, direito fundamental, serviço público,

\footnotetext{
${ }^{1}$ Este artículo es resultado del trabajo académico que se adelanta en la Línea de Estado, Democracia y Constitución del Grupo de Investigación en Conflicto y Paz (Categoría A1 - Colciencias) de la Facultad de Ciencias Sociales y Humanas de la Universidad de Medellín. La traducción del resumen es una contribución del profesor Rubén Palacio Mesa. Jefe del centro de idiomas y líder de la unidad de traducción de la Universidad de Medellín. rupalacio@udem.edu.co
} 
direito econômico, social e cultural, dignidade e autodeterminação.

\title{
Resumen
}

El presente texto tiene la finalidad de presentar las diversas expresiones socio-jurídicas y constitucionales en las que se ha concebido el derecho a la educación en Colombia. Esto supone un énfasis especial en la transición que se ha hecho de la educación como un servicio público o como un derecho prestacional a ser concebido y descrito como un derecho fundamental, más allá del orden lexicográfico. La razón para concebirse como derecho fundamental está condicionada por tres aspectos: el bloque de constitucionalidad; la interpretación expansiva del orden jurídico que reconoce la educación como un derecho fundamental no solo en favor de los niños y la realización de los principios de dignidad y autodeterminación. Para servir a este fin, primero se presentará una forma de entender las particularidades normativas que condicionan la categorización de un derecho como fundamental; luego, se situará la educación dentro de dicha concepción del derecho y, por último, se desarrollarán las principales tensiones que han surgido en el panorama político colombiano a partir de las acepciones que la Constitución de 1991 ha venido presentando históricamente del derecho a la educación y expresadas en dos sectores particulares de la interpretación jurisprudencial del derecho a la educación.

Palabras clave: educación, derecho fundamental, servicio público, derecho económico, social y cultural, dignidad y autodeterminación.

\begin{abstract}
The purpose of this text is to present the various socio-legal and constitutional expressions linked to how the right to education in Colombia has been conceived. This implies a special emphasis on the transition that has been made from education as a public service or as a right to a service, which is conceived and described as a fundamental right that goes beyond the lexicographic order. A fundamental right is conditioned by three aspects: (1) the constitutional block; (2) the expansive interpretation of the legal order that recognizes education as a fundamental right not only in favor of children and (3) the realization of the principles of dignity and self-determination. For the purpose of this text, a way of understanding the normative particularities that condition what the categorization of a fundamental right first be presented. Then, education will be placed within this conception of law. Finally, the main tensions that have arisen in the Colombian political landscape will develop from the meanings that the 1991 Constitution has historically presented the right to education and expressed in two particular sectors of jurisprudential interpretation of the right to education.
\end{abstract}

Keywords: education, fundamental right, public service, economic, social and cultural law, dignity and self-determination 


\section{INTRODUCCIÓN}

El derecho a la educación en Colombia ha recorrido un camino espinoso en lo que se refiere a su interpretación jurídica; y con ella, a la concreción de los medios fácticos para establecer su defensa y el aseguramiento de su protección en el escenario político. Desde el establecimiento de la Constitución Política de 1991 (Quinche, 2009, 45) el derecho a la educación se ha expresado como un servicio público, como un derecho social, económico y cultural (Arango, 2005, 37-45) y, también, como un derecho fundamental (Chinchilla, 2009, 91) llamado a defender principios y valores democráticos de primer orden como lo son la dignidad (Corte Constitucional, 2002, T-881), la igualdad (Corte Constitucional, 2016, T-276), y el acceso al conocimiento (Corte Constitucional, 2005, T-447).

La acepción que más tensión ha causado en los últimos tiempos la constituye la concepción por medio de la cual se comprende la educación como un derecho fundamental (Corte Constitucional, 1992, T-002), en la medida que en esta se encuentra un pilar de la vida política que repercute fuertemente en múltiples espectros, así, entender la educación como un derecho fundamental implica que el Estado en su agenda política está llamado a proveer los medios necesarios para el mantenimiento, dirección y control del sistema educativo colombiano, a su vez que establecer los mecanismos de su protección como, en el caso del texto constituyente, lo resulta ser la acción de tutela (Charry, 1992, 24).

Con esto, en lo que sigue se expresará dicha disputa que cobra sus gestas más relevantes en el terreno constitucional y que resulta determinante en el escenario de lo normativo a partir del cual se regula la legislación educativa del país. Sobre esta base de entendimiento, este texto se servirá del referente teórico de los derechos fundamentales, según se enmarcan en el sentido más normativo del lenguaje, esto es, según la consideración de que los sujetos pueden valerse de los presupuestos necesarios, materiales e inmateriales, para implantar su debida defensa y el aseguramiento de protección que se debe considerar en lo atinente a las garantías necesarias para la no vulneración del derecho mismo.

Con el fin de lograr este propósito, en un primer momento se presentará una reconstrucción de la estructura de un derecho fundamental, en sus aspectos normativos; las condiciones necesarias para que un derecho adquiera la categoría de fundamental y su distinción sustancial en lo que se refiere a categorías como la de los 
Derechos Humanos y las de servicios públicos y derechos sociales, económicos y culturales serán los principales ejes sobre los que girarán los argumentos a partir de los cuales se presentará esta concepción del derecho a la educación.

Posteriormente, se presentará una reconstrucción del entendimiento que en el sentido histórico se ha otorgado a la educación tanto en el marco del derecho internacional como en el contexto del derecho interno colombiano. Con esto se abrirá, pues, un round en esta contienda que tomará fuerza en la distinción particular que se ha tenido en Colombia en torno al tratamiento que se ha dado a la concepción de la educación como un servicio público y como un derecho social, económico y cultural frente a la concepción que la enmarca en los términos de compresión de un derecho fundamental.

Luego, se establecerán las razones jurídicas a partir de las cuales se expresa la educación como un derecho fundamental, por lo que, al tratarse de un pilar social, amparado por el Bloque de constitucionalidad (Estrada, 2007, 95; Corte Constitucional, 2016, C-327), que versa sobre la defensa de múltiples valores democráticos de primer orden como son la dignidad, la libertad, la igualdad, la autodeterminación y la supervivencia -por mencionar algunos- deben gozar de medios efectivos para el aseguramiento de su protección y el establecimiento de las garantías de aplicación sin condicionamientos de ningún tipo en el escenario jurídico.

Con todo esto, por último, se apartará un espacio para estimar dos de las principales interpretaciones que se han ofrecido, una a favor y otra en contra, de la discusión referente a los pros y los contras de la aplicación del Bloque de constitucionalidad en lo tocante a las libertades jurisdiccionales para el establecimiento de políticas educativas y para el desarrollo general del sistema educativo en el ejercicio del control estatal y de la autonomía del gobierno local en la implementación de los hilos orientadores de los programas educativos.

Se trata pues, en última instancia, del entendimiento de la educación -y no solo la educación básica y media, sino también la educación superior- como un valor universal y como un derecho fundamental que debe ser beneficiario de unos ciertos atributos, materiales e inmateriales, de los cuales es preciso se garantice la adecuada intervención del Estado. Y esto, por supuesto, no se piensa solo en términos de la ejecución de un control jurídico sobre la educación, sino también, del financiamiento y dotación adecuados del sistema educativo nacional, en lo que se refiere a cada una de las exigencias materiales, técnicas y humanas para que el debido acceso al 
conocimiento, a la ciencia, al arte y a la cultura se constituya en una realidad por medio de la efectiva inserción de las necesidades del sistema educativo en la agenda política colombiana.

\section{LOS DERECHOS FUNDAMENTALES. UNA CLARIDAD.}

En el marco normativo propio que se puede considerar dentro de un Estado Social de Derecho (Araújo, 2015, 358-360), los derechos fundamentales pueden ser fácilmente entendidos como aquellos que compendian los ideales del derecho subjetivo y las garantías jurídicas sobre las que cualquier sujeto puede asirse de un aparato normativo, fundado en la consagración de atributos morales o materiales, para exigir el aseguramiento de la ejecución y defensa de los principios de dignidad, supervivencia y autodeterminación. En principio, esta definición funda un cúmulo considerable de cuestiones que, cuando menos, deben ser aclaradas, y dentro de este, la principal claridad que cabe mencionar, para los fines de este texto, es la concerniente a la distinción que se puede encontrar de forma latente entre el lenguaje propio de los Derechos Humanos y el de los derechos fundamentales.

El discurso de los Derechos Humanos se incorpora dentro del habitus político universal a mediados del siglo XX sobre los cimientos de la corriente del iusnaturalismo (Radbruch, 2010,53) y tiene como bandera la defensa de principios irrenunciables y de carácter necesario para todos los individuos. Estos derechos son el resultado de la evidencia de la voluntad superior y establecen los condicionantes mínimos sobre los cuales son posibles la armonía y el equilibrio entre los individuos (Declaración Universal de los Derechos Humanos, 1948). Derechos de este tipo resultan exigibles en tanto se constituyen como un límite a la institucionalidad jurídica y son órganos de ejecución. Así enmarcados, los Derechos Humanos no pueden ser considerados sobre la categoría de una declaración resultante de una pugna en términos jurídicos sino como un atributo de las personas que constituyen en ellos su propia existencia y su valor en tanto individuos humanos (Pogge, 2005, 75).

Los derechos fundamentales, entonces, pueden establecerse sobre los términos de una concesión normativa en la que los sujetos pueden poner su acento para defender, exigir, reclamar o conceder una serie de atributos materiales e inmateriales que hagan posible la realización de la dignidad, la supervivencia o la autodeterminación (Restrepo, 2018, 57). 


\section{LA EDUCACIÓN EN EL DERECHO.}

Con lo anterior, resulta fácil aceptar la comprensión schilleriana a partir de la cual, por medio de la educación se puede acceder al saber y a la cultura (Schiller, 2000, 102), por ejemplo, en la medida que en el saber puede encontrarse una respuesta directa al cómo satisfacer todas aquellas necesidades de importancia vital en lo que se refiere al principio de supervivencia, pues en él se pueden considerar el desarrollo de los procesos de apropiación de las artes, los oficios y saberes técnicos que resultan ser un insumo importantísimo dentro de los que alimentan dicho principio. La educación es concebida como el medio a través del cual se asegura que las futuras generaciones tengan cabida fáctica y funcional en el seno de diversas sociedades (Paredes, 2016, 129)

Con este tipo de consideraciones, es menester hacer un breve hincapié respecto de la importancia de la distinción del régimen político particular del Estado del que se hable, ya que será un determinante importante en las formas de entendimiento y protección de los diversos sistemas educativos existentes en el panorama político del globo.

Así, en los Estados de un corte más liberal, el proyecto educativo ha tendido a fundarse en la idea de que la educación es un servicio público y, cómo tal, cada individuo puede acceder a ella o al nivel de ella que se puede costear por sus propios medios. En este sentido la educación y la calidad educativa no resultan ser factores que constituyan una preocupación determinante dentro de los programas de gobierno de países como Estados Unidos o Inglaterra, en la medida que, al ser considerados servicios públicos, están principalmente a cargo del sector privado (Lassalle, 2010, 235). En otro sentido, Estados con programas de gobierno de corte socialista ponen la totalidad de su apuesta por la educación en los principios rectores del Partido. Aquí la apuesta es por la no mercantilización de la educación; los procesos de enseñanza y de aprendizaje tienen como finalidad la erradicación del analfabetismo y la formación de los individuos para la vida política en los límites de su territorio. En un último sentido, los Estados de estirpe social-demócrata o, también, Estados sociales de derecho -Canadá, Alemania o Colombia, por ejemplo- presentan un entendimiento del proyecto educativo en una doble vía que considera tanto la concepción de lo privado como de lo público. En este tipo de Estados hay instituciones educativas que 
presentan todas las características mencionadas para el caso de los Estados liberales, pero, también se presentan instituciones educativas de carácter público en las que el Estado interviene para ofrecer un nivel de acceso a la educación de calidad a los individuos sin que el aspecto económico resulte ser un inconveniente sustancial al respecto de principios como la accesibilidad y la permanencia.

\section{LA EDUCACIÓN EN EL DERECHO COLOMBIANO.}

Pensar la historia del posicionamiento de la educación en el aparato jurídico colombiano implica recorrer un camino trazado por lágrimas y esperanzas en lo que se refiere al lugar que le ha otorgado el orden constitucional del país. Desde el establecimiento del documento magno con el que se reconfigura la dirección política colombiana, la Constitución Política de 1991, la educación es presentada y tratada desde el orden jurídico como un servicio público en la mayoría de sus ámbitos de ejecución, excepción hecha solamente en el caso de los niños, para quienes sí está establecida como un derecho fundamental (Artículo 44 Superior) aunque, como se podrá ver en lo que sigue, no tiene siquiera los medios de protección jurídica que por su definición en la Constitución deberían tener los derechos fundamentales.

En el ejercicio constituyente colombiano los derechos fueron enmarcados en tres categorías, a saber, en un primer orden, los derechos fundamentales; en un segundo orden, los derechos sociales, económicos y culturales y, por último; los derechos colectivos y medioambientales (Pérez, 2004, 291); para cada uno de estos, además se establecieron los medios jurídicos a partir de los cuales los individuos podrían establecer las garantías de su no vulneración. Así, se enmarcó el uso de la acción de tutela, destinando esta solo a la defensa particular de los derechos fundamentales que fueron concebidos como derechos de protección inmediata en el artículo 85 Superior. De esta manera, el particular artículo Superior delimitó la defensa y protección inmediata de los derechos fundamentales expresados solo entre los artículos 11 a 41 Superiores, por lo que un presupuesto lexicográfico excluiría a los niños de revestirse de la protección del velo tutelar. Con la necesidad de aclarar que por fuera de la lista de derechos fundamentales, sí reconoce en el artículo 44 Superior que los niños tienen derecho a la educación.

Ajustados literalmente a la voluntad de nuestro constituyente primario, la educación fue concebida tanto como servicio público y como derecho social, 
económico y cultural, de naturaleza prestacional y reducida, según el canon liberal al acceso de calidad según la posibilidad de pago; promoviéndose así una brecha de inequidad en el inmediato, mediano y largo plazo que resultaría incompatible con lo trazado en el marco dogmático y principialístico del Estado social de derecho (Cepeda, 2007, 96).

Es necesario advertir que por vía de interpretación constitucional, en cabeza de la Corte Constitucional (artículo 241 Superior), cuya función como guardián máximo e intérprete supremo del sentido constitucional, se debe ocupar de asegurar que exista una verdadera correspondencia entre la descripción normativa y la realidad social, ha señalado por vía de sus providencias (Sentencia T-002 de 1992; Sentencia T-743 de 2013; Sentencia T-008 de 2016 ) que la educación en sí misma, por su conexidad con la dignidad y la autodeterminación de cada sujeto ostenta la categoría de derecho fundamental, necesario y de importancia capital en la consolidación del conglomerado social y en el posicionamiento de los individuos en un escenario democrático, plural, deliberante, incluyente, participativo, crítico y generador de condiciones materiales e inmateriales a través de los cuales se asegura la realización de otros derechos básicos como la salud, el libre desarrollo de la personalidad, el trabajo, la asociación, la intimidad, la libertad de oficio, la participación ciudadana, el acceso a la administración de justicia y el respeto por los derechos de los demás, como fin en sí mismo (Kant, 2004, 139), más allá de su fe, su raza, su sexo o su filiación política.

Aquí es preciso analizar, punto por punto, el marco normativo que establece la Constitución para comprender el papel fundante de la educación en la sociedad colombiana; además, pensar los elementos reales y suficientes para concretar la necesidad de un cambio en el entendimiento de la esfera de derechos en la que se está ubicando la educación en el país.

Artículo 44 Superior reza así: "Son derechos fundamentales de los niños: la vida, la integridad física, la salud y la seguridad social, la alimentación equilibrada, su nombre y nacionalidad, tener una familia y no ser separados de ella, el cuidado y el amor, la educación y la cultura, la recreación y la libre expresión de su opinión. Serán protegidos contra toda forma de abandono, violencia física o moral, secuestro, venta, abuso sexual, explotación laboral o económica y trabajos riesgosos. Gozarán también de los demás derechos consagrados en la Constitución, en las leyes y en los tratados internacionales ratificados por Colombia. La familia, la sociedad y el Estado tienen la 
obligación de asistir y proteger al niño para garantizar su desarrollo armónico e integral y el ejercicio pleno de sus derechos. Cualquier persona puede exigir de la autoridad competente su cumplimiento y la sanción de los infractores. Los derechos de los niños prevalecen sobre los derechos de los demás." (Cursiva fuera del texto original)

Artículo 67 Superior reza así: "La educación es un derecho de la persona y un servicio público que tiene función social; con ella se busca el acceso al conocimiento, a la ciencia, a la técnica, y a los demás bienes y valores de la cultura. La educación formará al colombiano en el respeto a los derechos humanos, a la paz y a la democracia; y en la práctica del trabajo y la recreación, para el mejoramiento cultural, científico, tecnológico y para la protección del ambiente. El Estado, la sociedad y la familia son responsables de la educación, que será obligatoria entre los cinco y los quince años de edad y que comprenderá como mínimo, un año de preescolar y nueve de educación básica. La educación será gratuita en las instituciones del Estado, sin perjuicio del cobro de derechos académicos a quienes puedan sufragarlos. Corresponde al Estado regular y ejercer la suprema inspección y vigilancia de la educación con el fin de velar por su calidad, por el cumplimiento de sus fines y por la mejor formación moral, intelectual y física de los educandos; garantizar el adecuado cubrimiento del servicio y asegurar a los menores las condiciones necesarias para su acceso y permanencia en el sistema educativo. La Nación y las entidades territoriales participarán en la dirección, financiación y administración de los servicios educativos estatales, en los términos que señalen la Constitución y la ley." (Cursiva fuera del texto original).

Artículo 68 Superior reza así: "Los particulares podrán fundar establecimientos educativos. La ley establecerá las condiciones para su creación y gestión. La comunidad educativa participará en la dirección de las instituciones de educación. La enseñanza estará a cargo de personas de reconocida idoneidad ética y pedagógica. La ley garantiza la profesionalización y dignificación de la actividad docente. Los padres de familia tendrán derecho de escoger el tipo de educación para sus hijos menores. En los establecimientos del Estado ninguna persona podrá ser obligada a recibir educación religiosa. Los integrantes de los grupos étnicos tendrán derecho a una formación que respete y desarrolle su identidad cultural. La erradicación del analfabetismo y la educación de personas con limitaciones físicas o mentales, o con capacidades excepcionales, son obligaciones especiales del Estado." (Cursiva fuera del texto original). 
Artículo 69 Superior reza así: "Se garantiza la autonomía universitaria. Las universidades podrán darse sus directivas y regirse por sus propios estatutos, de acuerdo con la ley. La ley establecerá un régimen especial para las universidades del Estado. El Estado fortalecerá la investigación científica en las universidades oficiales y privadas y ofrecerá las condiciones especiales para su desarrollo. El Estado facilitará mecanismos financieros que hagan posible el acceso de todas las personas aptas a la educación superior." (Cursiva fuera del texto original)

Artículo 70 Superior reza así: "El Estado tiene el deber de promover y fomentar el acceso a la cultura de todos los colombianos en igualdad de oportunidades, por medio de la educación permanente y la enseñanza científica, técnica, artística y profesional en todas las etapas del proceso de creación de la identidad nacional. La cultura en sus diversas manifestaciones es fundamento de la nacionalidad. El Estado reconoce la igualdad y dignidad de todas las que conviven en el país. El Estado promoverá la investigación, la ciencia, el desarrollo y la difusión de los valores culturales de la Nación." (Cursiva fuera del texto original)

Todas estas disposiciones normativas dan cuenta de la importancia de la educación en el escenario social e individual del país. Pero aun con esto, el problema funcional del entendimiento de la educación en estos términos radica, propiamente, en las limitadas dimensiones de su exigibilidad, en tanto que las posibilidades de su aplicación y protección se encuentran siempre condicionadas a las posibilidades fácticas asistenciales de la agenda pública, con lo que se ven comprometidos los principios constitucionales de primer orden como la dignidad, la supervivencia y la autodeterminación.

Resulta de una importancia capital entender el léxico constitucional para pensar las posibilidades fácticas de una transición que se presenta aquí como necesaria de la concepción inicial de la educación como un derecho social, económico y cultural a la categoría plena de derecho fundamental susceptible de protección por la vía de la acción de tutela. A este respecto, se debe establecer que el requisito primario para que se le pueda ofrecer la categoría de derecho fundamental a un derecho, es que ocurra una declaración explícita que enuncie dicha condición por parte de un órgano normativo (Bloque de constitucionalidad, Constitución, ley, decreto o sentencia). La declaración expresa del derecho y su categorización de fundamental es, en última instancia, lo que le da existencia y le posibilita la exigibilidad jurídica.

La Corte Constitucional ha jugado un papel muy importante en este punto 
porque se ha valido de una interpretación garantista (Ferrajoli, 2011, 365) con respecto al sentido pro homine, a la hora de adoptar como propias, medidas derivadas de la legislación internacional en la que señala que donde dice niño debe leerse toda persona. Es así como la educación goza de una doble condición. En cuanto al carácter literal de la Constitución es un servicio público y un derecho prestacional; que solo aplica como derecho fundamental en favor de los menores. Pero al tomar la posición garantista y vinculante que ocurre en la normativa internacional, la Corte Constitucional acepta que existe un nexo causal entre la educación y los principios de dignidad y de autodeterminación; de tal manera que extiende el sentido interpretativo del derecho a la educación como derecho fundamental en favor de todos los asociados. Un derecho fundamental autónomo y susceptible de protección mediante acción de tutela (Sentencia T-434 de 2018).

\section{LA CONSTITUCIÓN DE 1991 FRENTE AL DERECHO INTERNCIONAL.}

Una vinculación importante del lenguaje constitucional colombiano se encuentra expresado en los artículos 93 y 94 Superiores, en los que se inserta el Bloque de constitucionalidad (Suelt-Cock 2016, 310); concebido como el conjunto de tratados, principios y pactos internacionales que consagran una amplia lista de derechos, que al ser ratificados por el Congreso, se aplican de manera preferente en el orden interno (Artículo 93 Superior). Asimismo, establece que los derechos innominados o los tratados que no están ratificados pero que se encargan de la defensa o promoción de alguna esfera particular considerada como esencial para asegurar la supervivencia, dignidad o autodeterminación de un sujeto, deben acoplarse de igual forma en el uso y marco de interpretación normativa que, de igual manera, también opera de manera vinculante y decisiva en el orden interno (artículo 94 Superior).

Los tratados internacionales que materializan una defensa del derecho a la educación como un derecho que ofrece las medidas para la humanización de los individuos y de preservación de los principios democráticos tienen, por definición, un lugar preponderante en la agenda política, legal y social de Colombia. Para contrastar la expresión de esta concesión normativa frente a los principios expresados en el marco internacional, es pertinente considerar aquí algunos de dichos tratados y los alcances de su establecimiento. 
Declaración Universal de los Derechos Humanos (1948, Artículo 26): en este importante artículo se deja la declaración expresa de que la educación es un derecho de todas las personas; se establece, además, que debe ser gratuita; que la instrucción básica de las personas del globo debe ser obligatoria y, más importante aún, que el objeto de la educación no es otro que el desarrollo integral de la persona y su formación en el respeto por los derechos humanos.

\section{$\checkmark$ Pacto de Derechos Económicos, Sociales y Culturales (1966, Artículo 13):} concibe la educación como un derecho del que deben gozar todos los individuos del género humano y su finalidad es permitir el libre desarrollo de las personas y formarlas para la participación en sociedades libres, democráticas y plurales sobre el principio de preservación y protección de los derechos humanos.

$\checkmark$ Convención sobre la eliminación de todas las formas de discriminación contra la mujer (1979, Artículo 10): se establece el deber de garantizar que todas las personas, hombres y mujeres sin distinción, accedan a una educación cualificada y que mantenga el principio de igualdad de oportunidades en lo que se refiere al acceso al conocimiento y a los debidos medios para asegurar la participación en modelos de enseñanza pertinentes y equitativos, sin ningún tipo de distinción.

\section{$\checkmark$ Declaración sobre los derechos de los pueblos indígenas (2007, Artículo} 14): expresa la libertad de la que gozan todas las comunidades indígenas para establecer sus formas de institucionalidad educativa y fijar sus propios mecanismos de control sobre la misma; entendiendo la importancia de la preservación de sus lenguas y garantizando un acceso igualitario de inserción a la sociedad en términos de oportunidades.

Sobre esta base de entendimiento puede decirse, hasta aquí, que este tipo de acuerdos internacionales han aportado a la justificación de la necesidad imperante de una transición en el entendimiento de la educación como un derecho que da cuenta de la concepción de una condición necesaria para potenciar las facultades humanas que posibilitan el fortalecimiento del establecimiento democrático de las naciones que se amparan en la máxima del ideal de los derechos humanos.

\section{EL BLOQUE DE CONSTITUCIONALIDAD PUESTO EN DISCUSIÓN.}

La injerencia política de instituciones internacionales en materia educativa es un cuento de vieja data. El esfuerzo político-institucional de ciertas entidades ha 
direccionado y regulado la política institucional en la región, un hecho que es aplaudido por amplios sectores y reprochado por otros. Los primeros señalan la importancia de que los discursos educativos adquieran cierta unidad administrativa, que la forma en que se entienda la educación atienda a necesidades globales como la dignificación de la vida del hombre, la movilidad social y la investigación científica; los segundos arguyen que esta centralización de la educación ha comprometido la jurisprudencia de los Estados para construir, per se, un andamiaje educativo que se justifique por sus propias realidades político-sociales; más aún, que los proyectos educativos obedezcan a intereses económicos que favorecen al mercado neoliberal, instrumentalizando a los Estados y a la educación misma, condenándola a lógicas clientelistas.

En Colombia esta influencia adquiere también una doble connotación. Por un lado, la influencia política de entes transnacionales como la ONU y su compromiso con la articulación de los Derechos Humanos ha promovido medidas normativas y organismos institucionales que han garantizado la salvaguarda de la educación, entendida como un derecho fundamental por el léxico y la historiografía constitucional colombiana. Esta translación hermenéutica de la educación como derecho humano básico en el ámbito internacional, a derecho fundamental a nivel local, ha enriquecido las garantías políticas del fenómeno educativo, brindando un reconocimiento que, a la luz del Estado social de derecho (Sentencia T-406 de 1992), se ha ajustado a los ideales de formación política de la ciudadanía del país. Por otro lado, la educación como servicio ha permitido el establecimiento de una oferta educativa caracterizada por generar escenarios de aprendizaje que se ajusten al interés de formación de los propios ciudadanos, en relación de su capacidad adquisitiva, principios políticoreligiosos o educativos.

Ahora, es necesario indagar de qué manera conviven ambas connotaciones normativas. Constitucionalmente, Colombia se compromete a incorporar el léxico de los Derechos Humanos a partir del Bloque de constitucionalidad, lo que implica que se reafirme la educación como un compromiso político por garantizar el status de ésta como principio fundamental de la vida digna, el acceso a las oportunidades según el mérito o las limitaciones, la convivencia pacífica y la movilidad social. Esta aseveración, que también representa una lucha contra la pobreza, el desarrollo y la formación del ser humano, amplía la garantía de su cumplimiento, elevando a un carácter de obligatoriedad y gratuidad en la educación primaria y secundaria, y 
estableciendo un compromiso con la accesibilidad a la educación superior.

Es precisamente a raíz de la última idea donde se genera la dualidad que aquí se pone en cuestión. La educación es comprendida como derecho toda vez que el Estado afirma que allí se debaten formas y expresiones indisolubles e indisociables a la dignidad y la subsistencia humana, por lo cual es necesario brindarle los debidos medios políticos para su reivindicación, tal como la tutela; por otro lado, el Estado asume un rol regulador y garante cuando adquiere el carácter de servicio público que puede ser sustentado y prestado por los propios ciudadanos según un ánimo de lucro.

La dualidad con que se entiende la educación en el país no es un asunto meramente nominal. La discusión política en torno a la educación como derecho y servicio transversaliza todo el andamiaje institucional, normativo y pedagógico. Desde elementos cotidianos de la vida en las aulas como la evaluación y los formatos de sistematización de experiencias educativas, hasta elementos relacionados con la cobertura y los criterios de promoción y evaluación (Runge, 2019, 60).

Así, mientras la tipificación de la educación como derecho fundamental ha provocado que la educación básica y media goce de cierta cobertura en instituciones oficiales, la educación superior adolece de recursos fácticos, técnicos, logísticos y administrativos; con lo que se da la aparición de serias dificultades estructurales por cuenta de la desigualdad socio-económica de un país donde no todos pueden acceder a una educación con calidad. Estas y otras dificultades acompañan la cotidianidad en el aula, a las que se suma la falta de inversión en instalaciones físicas, la poca presencia institucional en zonas rurales del país o la falta de programas de formación para maestros y estudiantes. En este horizonte de sentido, el compromiso del Estado parece ser engullido por las lógicas de financiamiento de una educación que, tal como lo plantean los discursos empresariales o mercantiles, prestan su servicio en función de intereses más o menos privados, incluso, en escenarios oficiales. Esta falta de personificación de la educación, que se agudiza en la educación superior se marca por razones afines al mercado, a la creciente especulación laboral y la falta de financiación de las universidades públicas, que tuvieron por mucho tiempo una relación atemporal con el presupuesto destinado para su debido funcionamiento.

Naturalmente la dicotomía de la educación entendida como servicio y como derecho fundamental se vuelca sobre la disyunción fáctica que esta genera en razón de la realidad institucional y normativa de un derecho que se agita entre la cobertura de la educación básica y media y el aseguramiento de las garantías institucionales y 
normativas para la educación superior. Más allá de esto, es clave señalar que como derecho fundamental y como servicio público, el proyecto educativo de país es un proyecto, entre otras cosas, inacabado.

\section{A manera de conclusión.}

La educación en Colombia es entendida como un servicio público, como un derecho social y también, por declaración expresa del máximo intérprete constitucional, como un derecho fundamental de orden primario en lo tocante a la vida en sociedad, por parte de los individuos y frente a los demás asociados. La educación se erige como principal garante social del afianzamiento de los valores democráticos y el relacionamiento de las personas dentro de una sociedad libre, horizontal, plural y deliberante. Se supera la concepción básica de servicio público o derecho social económico y cultural, limitado a posibilidades fácticas de inversión por parte del Estado o a la capacidad de financiación de cada sujeto.

Declarar la educación como derecho fundamental significa asistir a un uso fáctico y hermenéutico del bloque de constitucionalidad que muestra la conexidad entre dignidad y autodeterminación con los presupuestos básicos de enseñanza y aprendizaje que se desarrollan en el interior del sistema educativo; de igual manera se pone en evidencia de qué manera la Corte Constitucional realiza una interpretación expansiva de la normativa internacional para afirmar que la condición de derecho fundamental no se aplica de manera exclusiva y excluyente frente a los niños sino que debe cobijar a todas las personas. A cada una, según sus posibilidades, necesidades y el momento histórico en el contexto de la cultura, el mercado, la raza y las creencias religiosas.

La tipificación particular de la educación colombiana como derecho fundamental, en favor de todos, ha generado que el sistema educativo del país se vea seriamente afectado, en tanto, mientras las instituciones de educación básica y media gozan de los beneficios que le son otorgados por su reconocimiento institucional, la educación superior pública padece diariamente su condición prestacional que ha generado un gran cúmulo de dificultades de todo tipo, movilizadas principalmente por la desigualdad económica y social del país y que se traducen, en última instancia, en la falta de inversión en infraestructura, en ausencia de Estado y en la oferta académica precaria para atender necesidades particulares de la población asentada en zonas 
periféricas.

Es deber estatal de asegurar la satisfacción de condiciones materiales e inmateriales que posibiliten que todo el sistema educativo colombiano, en todos sus niveles, está erigido sobre los principios democráticos que facultan a los individuos para la vida en sociedad, para el respeto por los Derechos Humanos, propios y ajenos, y para el acceso a los saberes particulares que posibiliten la satisfacción del principio de supervivencia y la calidad de vida de los asociados y su entorno.

El accionar estatal frente a la educación, concebido como derecho fundamental, debe tomarse en serio la realización de acciones que garanticen financiación, vigilancia y control de políticas públicas que provean parámetros de calidad, progreso social, investigación aplicada, defensa de la identidad cultural y desarrollo de la personalidad individual, enmarcados en la defensa y promoción de los valores democráticos de diversidad, multiperspectivismo y solución pacífica de conflictos.

Son muchas las instituciones internacionales que han puesto sus mecanismos de aplicación del derecho al servicio de la educación y su categorización en el ámbito democrático como un Derecho Humano que encuentre su transliteración en el escenario normativo local como un derecho fundamental. Este esfuerzo políticoinstitucional ha representado múltiples discusiones que en Colombia han cobrado gran protagonismo en el tratamiento constitucional que se ha dado a este derecho. Por un lado, grandes sectores de relevancia en el panorama constitucional colombiano hacen hincapié en que los discursos educativos se integren en la expresión internacional de la necesidad de una cierta unidad discursiva que presente la educación como un estamento articulador de la vida humana, llamada a defender principios de tamaño alcance como la dignidad, la movilidad social, la promoción de la cultura democrática, la apropiación de la ciencia y la formación integral de cada sujeto. Por otra parte, se presentan en el panorama político colombiano otros sectores que ponen el acento de la discusión en las posibles repercusiones que podría traer consigo la adopción de discursos que, según expresan, apuestan por la centralización de la educación. Según estos sectores, la apropiación del discurso educativo expresado por las instituciones internacionales tienen la finalidad de consolidar sistemas educativos que favorezcan los intereses particulares del mercado neoliberal, en tanto se fundan en la pretensión de instrumentalizar a los Estados y a sus sistemas educativos, con lo que se pone en riesgo la injerencia de los Estados locales en el establecimiento de las políticas 
educativas y se quita el acento puesto en las realidades y necesidades políticosociales particulares del sistema educativo de la región.

La inclusión del discurso de los Derechos Humanos, promovido por instituciones internacionales, abre las posibilidades para la conformación de medidas normativas e institucionales de salvaguarda necesarias para la educación dentro de los escenarios políticos locales, lo que representa una garantía de reconocimiento en lo que se refiere al papel fundante de la educación en lo atinente a la formación política de la ciudadanía.

El entendimiento de la educación como servicio público y como un derecho social, económico y cultural ha posibilitado concretar el establecimiento de una oferta educativa que ha puesto el acento sobre las necesidades de formación de los colombianos en consideración a sus posibilidades económicas y al establecimiento de proyectos educativos contextualizados en lo tocante a la formación operativa e instrumental del individuo que cualifica su fuerza de trabajo.

La suerte de dualidad sobre la cual se ha entendido el derecho a la educación en Colombia no la constituye un ejercicio meramente nominal, sino que la específica categorización del derecho a la educación establece una vía de aplicación transversal a todo el modelo institucional de la educación nacional en sus ámbitos de desarrollo. Así, se ven afectados los momentos primarios mismos del quehacer educativo, como son los mecanismos de evaluación, los programas de cobertura y los criterios de promoción. La disyuntiva sobre la cual se presenta expresado el derecho a la educación en Colombia, en la medida que da muestra de la diferencia contundente existente entre los niveles de cobertura de la educación básica y media, en relación a las problemáticas que aguzan a la educación superior del país, agudizadas por esta disputa en los últimos años, deja en claro que la educación en Colombia toma la forma de un proyecto inconcluso, que no encuentra su total sentido orientador debido a la falta de los recursos jurídicos, materiales e inmateriales, para asegurar su efectiva salvaguarda desde el órgano estatal.

\section{REFERÊNCIAS}

Araujo, J. (2015). Filosofía o teoría del derecho constitucional. Bogotá: Ibáñez. 
Arango, R. (2005). El concepto de derecho sociales fundamentales. Bogotá: Legis.

Cepeda, J. (2007). Polémicas constitucionales. Bogotá: Legis.

Constitución Política de Colombia, (2019). Bogotá: Temis.

Charry, J. (1992). La acción de tutela. Bogotá: Temis.

Chinchilla. T. (2009). ¿Qué son y cuáles son los derechos fundamentales? Bogotá: Temis.

Corte Constitucional de Colombia, (2018). Sentencia T-434 de 2018.

Corte Constitucional de Colombia, (2016). Sentencia C-327 de 2016.

Corte Constitucional de Colombia, (2016). Sentencia T-276 de 2016.

Corte Constitucional de Colombia, (2016). Sentencia T-008 de 2016.

Corte Constitucional de Colombia, (2013). Sentencia T-743 de 2013

Corte Constitucional de Colombia, (2005). Sentencia T-447 de 2005.

Corte Constitucional de Colombia, (2002). Sentencia T-881 de 2002.

Corte Constitucional de Colombia, (1992). Sentencia T-406 de 1992.

Corte Constitucional de Colombia, (1992). Sentencia T-002 de 1992.

Declaración sobre los derechos de los pueblos indígenas, (2007).

Declaración Universal de los Derechos Humanos, (1948).

Estrada, S. (2007). Los principios jurídicos y el bloque de constitucionalidad. Medellín: Sello editorial de la Universidad de Medellín.

Ferrajoli, L. (2011). Principia luris. Teoría del derecho y de la democracia. Tomo 2. Teoría de la democracia. Madrid: Trotta.

Kant, I. (2004). Fundamentación de la metafísica de las costumbres. Madrid: Alianza.

Lassalle, J. (2010). Liberales. Compromiso cívico con la virtud. Barcelona: Debate Muñoz, V. (2006). Prólogo. Un enfoque de la educación basado en los derechos humanos. Nueva York. EEUU. UNICEF.

Pacto de Derechos Económicos, Sociales y Culturales, (1966).

Paredes, M. (2016). Educación democrática. La propuesta de John Dewey. Bogotá: Editorial aula de humanidades. 
Pérez, J. (2004). Derecho constitucional colombiano. Bogotá: Temis.

Pogge, T. (2005). La pobreza en el mundo y los derechos humanos. Barcelona: Paidós.

Quinche, M. (2009). Derecho constitucional colombiano. De la carta de 1991 y sus reformas. Bogotá: Universidad del Rosario.

Radbruch, G. (2010). Introducción a la filosofía del derecho. México: FCE.

Restrepo, J. (2018). Estructura constitucional del estado colombiano. Medellín: Sello editorial Universidad de Medellín.

Runge, A. (2019). Culturas escolares, culturas docentes y profesionalidad pedagógica. Medellín: Secretaría de educación de Antioquia.

Schiller, J. (2002). Escritos sobre estética. Barcelona: Tecnos.

Suelt-Cock, V. (2016). El bloque de constitucionalidad como mecanismo de interpretación constitucional. Aproximación a los contenidos del bloque en derechos en Colombia. Vniversitas. Bogotá (Colombia) № 133: 301-382, juliodiciembre de 2016. 